\title{
DER DICHTER JOHN AUDELAY UND SEIN WERK.
}

Wenn ich hier das wichtigste tuber John Audelay und seine gedichte veröffentliche, so geschieht es mit der bitte an die fachgenossen, mir uber ähnliche, weniger bekannte gedichte, die Audelay als quellen benutzt haben könnte, mitteilungen zu machen, da es mir sonst bei der grossen zahl der gedichte kaum möglich sein wtrde, ihre herausgabe fur die Early English Text Society mit quellenangaben in absehbarer zeit fertig zu stellen.

John Audelay ist kein grosser dichter, und wenn er in unserer zeit lebte, wurde er wohl kaum der beachtung wert gehalten werden. Da er aber schon im 15. jahrhundert lebte und seine religiösen gedichte verfasste, und da uns diese in einer leidlicb guten handschrift uberliefert sind, da er ausserdem selbst den ort nennt, wo er lebte und dichtete, und wir daher annehmen dürfen, dass die sprache seiner gedichte uns die mundartlichen eigenheiten dieser gegend zeigt, so sind wir nicht allein genötigt, seine erzeugnisse einer wissenschaftlichen untersuchung zu unterziehen, sondern mögen auch geradezu froh sein, dass uns ein gutiges geschick dieses buch erhalten hat. Müssen wir doch für jeden uns erhaltenen rest des mittelalterlichen lebens dankbar sein, mag er auch in hinsicht auf ästhetische schönheit fur unseren geschmack noch so viel $\mathrm{zu}$ wunschen ubrig lassen.

Die gedichte des John Audelay sind uns in der handschrift Donce 302 in der Bodleyschen bibliothek zu Oxford erhalten; Dr. Furnivall, der vorsitzende der Early English Text Society, hat mich mit ihrer heransgabe fur diese gesellschaft betrant. Im sommer 1889 habe ich die handschrift zu Oxford abge- 
schrieben; sie ist nicht gerade besonders leserlich. Fruher scheint sie einem Mr. R. Farmer gehört zu haben, der auf dem vorsatzblatte folgendes eingetragen hat:

"I have not found any Account of this | old \& blind Poet, John Audelay: | He calls himself Capellanus. His Work consists of metrical Divinity, |\& pious Legends - $S^{t}$ Gregory, $S^{t}$ Brigitt, | \&c. \&c."

- Die handschrift ist ein dunner quartband und besteht ans 35 pergamentblättern; jede seite ist in zwei spalten beschrieben.

Bisher ist von den gedichten Audelays nur etwa ein drittel von James Orchard Halliwell 1844 fur die Percy Society (als 14. band) herausgegeben worden, - eine ausgabe, die das sei nebenbei bemerkt - sehr viel za wthnschen thbrig lässt. Ich will hier nur zwei fehler im vorubergehen erwähnen, die zu den schlimmsten gehören: Eine der tberschriften bringt die bibelstelle: 'Averte oculos tuos, ne videant vanitatem. Halliwell druckt statt dessen: 'Ante oculos tuos ne videant vanitatem', was natürlich ganz sinnlos ist. Die handschrift hat deutlich Aũte, Halliwell hat also das häkchen tbersehen und blindlings 'Ante' gedruckt. Einige zeilen weiter heisst es: 'they likon hym to a lollere and to an epocryte'. Was aber hat Halliwell gelesen? - : 'to a lossere'!! - Ausserdem ist nur noch das gedicht einmal gedruckt worden, das das gesicht des heiligen Paul ('Visio Sancti Pauli' oder 'Die 11 Höllenstrafen') behandelt, und zwar von Morris im anhange vom 'Old English Miscellany' (1872, Early English Text Society).

John Audelay ist in folge dessen wenig bekannt, ich habe ihn nur bei den folgenden litteraturgeschichtschreibern erwähnt gefunden :

1) Morley spricht tiber ihn in seinen 'Illustrations of English Religion' auf s. 113; er beschreibt dort den gegensatz zwischen den Lollarden und ihren gegnern im 15. jahrhundert und sagt: 'The question is all of Duty; and from a quiet, orthodox monk, who was no great genias, though he wrote verse, but was a good natural Englishman, we may learn how thousands of honest folk, who took no violent part in the strife, looked at each side of it.' Dann folgen wenige zeilen uber den dichter und den inhalt seiner gedichte und einige ausztige aus diesen. 
2) Miss Jackson erwähnt unseren dichter in ihrem 'Glossary of the Shropshire Dialect' und giebt einige seiner mundartlichen eigentumlichkeiten.

3) Eine kurze schilderung Audelays und seiner werke findet sich auch in Brandls 'Mittelenglischer Litteraturgeschichte' in Pauls Grundriss (II. 704).

Mag der dichter auch noch anderswo erwähnt sein, jedes falles werden sich die bemerkungen auf die Halliwellsche ausgabe stlutzen; diese aber giebt nur ein bruchstiuck des ganzen werkes, allerdings auch das wichtigste des ubrigen textes in ganz kurzen auszitgen.

Sehen wir nun zunächst, was wir uber des dichters leben aus seinem buche - denn anderswo können wir nichts uber ihn finden - erfahren.

Das wichtigste steht in der zweiten hälfte der gedichtsammlung hinter der 4349. zeile; dort ist mit roter tinte folgendes geschrieben:

„Finito libro : sit laus \& gloria Christo | liber vocatur : concilium conciencie sic nominatur|Aut scala celi : \& vita salutis eterni | Iste liber fuit compositus per Johannem Awdelay | capellanum . qui fuit secus [= cæcus] \& surdus in sua | Visitacione . Ad honorem domini nostri Jesu Christi . \& \& ad exemplum aliorum in monasterio | de haghmon. Anno domini millesimo $\operatorname{ccc}^{\text {mo }} \mid$ visecimo vi $^{\text {to }}$ cuius anime propicietur deus Amen."

Hiernach war also Joln Audelay kaplan im kloster Haghmon(d), das nach der Miss Jackson 'Glossary' vier meilen nordöstlich von Shrewsbury lag; er war blind und taub; um das jahr 1426 schrieb er seine gedichte.

Dass die handschrift nicht vom dichter selbst geschrieben sein kann, ist natturlich, da er ja blind war; wohl aber ist möglich, und auch sehr wahrscheinlich, dass sie nach seinem eigenen diktat geschrieben wurde; sie stammt nämlich nach angabe des 'Douce Catalogue' aus dem anfange des 15. jahrhunderts, und nach der versicherung des unterbibliothekars der Bodleyschen bibliothek, Mr. Madan, aus den jahren zwischen 1430 und 1440. Mr. Madan meinte, der verfasser mulsse tot gewesen sein, als die handschrift angefertigt wurde, was aus der letzten zeile des eben angefuhrten absatzes hervorgehe, die auch am schlusse des ganzen bandes hinter der 6657. zeile 
wiederholt wird: 'Cujus anime propicietur Deus'. Möglich ist das ja, und wir würden also dann annehmen müssen, dass Audelay etwa 1430 gestorben, und dass dann diese handschrift nachher geschrieben worden sei, was sich ja auch mit den anderen angaben vereinigen lässt. Ebensogut möglich dunkt es mich aber anzunehmen, dass die handschrift noch bei lebzeiten des verfassers nach seinem eignen diktat angefertigt wurde, und dass der schreiber, voll achtung vor dem gottesfurchtigen, blinden dichter, den frommen wunsch am schlusse einiger gedichte anbrachte, „dass gott seiner seele gnädig sein möge." Weshalb soll dieser wunsch nicht schon bei lebzeiten des bedachten ausgesprochen werden können? - Was mich aber noch hauptsächlich zu der annahme drängt, dass grade die uns vorliegende einzige handschrift unmittelbar nach dem diktat des dichters geschrieben worden sei, ist der umstand, dass sie eine ausserordentlich grosse anzahl von unverständlichen stellen sowie von verbesserungen und veränderungen enthält, die ich mir eben dadurch erkläre, dass der schreiber dem diktat des dichters, der wegen seiner taubheit vielleicht nicht grade deutlich sprach, so gut folgte wie es ihm eben möglich war, aber manche worte nun doch nicht sogleich verstand und für sie eben hinschrieb, was er gehört zu haben glaubte. Durch die veränderungen und verbesserungen hat er dann nachher versucht, das undeutliche klar zu stellen. Vielleicht starb der dichter kurz nach vollendung der handschrift, so dass es dem schreiber nicht mehr möglich war, sie ihm noch einmal vorzulesen und dann genauer zu machen.

Was können wir nun noch aus den gedichten selbst uber des dichters leben etwa lernen?

In den letzten beiden strofen vor der erwähnten anmerkung uber Audelays leben finden wir einige angaben, die der dichter noch mehrmals am ende von anderen gedichten wiederholt; es heisst dort:

4324 As I lay seke in my langure

In an abbay here be west, pis boke I made with gret dolour,

When I myzt not slep ne haue no rest;

Oft with my prayers I me blest

\& sayd hile to heuen kyng:

4330 "I knowlache, lord, hit is pe best

Mekele to take pi vesetyng; 
Ellis wot I wil pat I were lome.

of al lordist be he blest!

Fore al pat ze done is fore pe best,

Fore in pi defawte was neuer mon lost

pat is here of womon borne.

Meruel ze not of pis makyng,

Fore $I$ me excuse hit is not $I$,

pis was pe hole gost wercheng,

4340 pat sayd pese wordis so faypfully;

Fore I quop neuer bot hye foly;

God hap me chastyst fore my leuyng.

I pong (= ponk) my god my grace treuly

For his gracious vesityng.

Beware, seris, I zoue pray;

Fore I mad pis with good entent

In pe reuer[en]s of God omnipotent;

4349

Prays fore me pat bep present.

Ganz ähnlich erscheint die zweite dieser strofen auch schon vorher (3202 ff.), und öfter.

Seinen namen nennt der dichter ferner noch: 1270, 1377, 2702, 2792, 2914, 3214, 3578, 3847, 5004, 5131, 5628, 6071, 6129,6644 ; einige male in verbindung mit der bitte um christliche furbitte fur seine seele; zuweilen auch mit beiwörtern wie synful und blynd.

Endlich sei hier noch die schluss-strofe der ganzen sammlung herangezogen:

6645 No mon pis book he take away

Ny kutt owte noo leef - y say forwhy -

For hit ys sacrelege, sirus, y zow say,

Bep acursed in pe dede truly!

3ef ze w[i]l haue any copi,

6650 Askus leeue \& ze shul haue;

To pray for hym specialy

That hyt made zour soules to saue,

Jon pe blynde Awdelay;

The furst prest to pe lord Strange he was

Of pys chauntre her in pis place,

That made pis bok by goddus grace,

Deeff, siek, blynd as he lay.

Cuius anime propicietur deus."

Leider - das will ich gleich hier bemerken - hat sich die nachwelt nicht an seinen fluch gekehrt, denn die ersten, 
etwa zehn, blätter fehlen und auch zwischen dem siebenten und dem achten vorhandenen blatte ist eine litcke, eine dritte zwischen dem 19. und dem 20.; zwei andere, die ich anfangs vermutete, sind nur scheinbar vorhanden.

Viel ist es also nicht, was wir so uber unseren dichter aus seinen werken erfahren; wir mussen das andere zwischen den zeilen lesen, und da ergiebt sich denn mit einiger sicherheit, dass unser guter Audelay in seiner jugend viel mitgemacht hat, vielleicht gar ein rechter lebemann gewesen ist, dass er dann aber in seinem alter, mit blindheit und taubheit geschlagen, in der stillen klosterzelle voll bitterer reue auf sein vergangenes leben zurtuckblickt und nun die sünden seiner jugend durch die grösste frömmigkeit wieder gut zu machen sucht, indem er das lob gottes und der heiligen singt und andere vor liederlichem lebenswandel warnt und an ihre pflichten gemahnt. In Stephens' 'Dictionary of National Biography' heisst es von ihm: „Judged by his writings he seems to have been of an unworldly and devout character." Die ungläubigen Lollarden verachtet er, rtigt mit grosser schärfe die ansichten Wiclifs und warnt vor ihnen, indem er die ewige verdammnis als lohn der ketzerei verkundet. So kommen wir zu dem inbalte von Audelays gedichten.

Den ersten und - auch ohne bertucksichtigung der lucken - grösseren teil seiner gedichte fasst Audelay selbst, wie wir sahen, unter dem namen zusammen: „Der Rat des Gewissens; die Himmelsleiter; oder Das Leben des ewigen Heils“. Er besteht aus einer anzahl verschiedener gedichte, die durch besondere uberschriften kenntlich gemacht sind, auch je verschiedenes versmass haben.

\section{Erste abteilung (1-4349).}

Erstes gedicht (1-262).

Das erste gedicbt ist bruchstlick; es muss ursprtinglich das zehnte der ganzen sammlung gewesen sein, denn uber der zweiten spalte der vorderseite des zweiten blattes, in der bei 263 das zweite vorhandene gedicht beginnt, steht: ๆ XI. - Die erste litcke ist also recht gross; das geht auch aus einer bemerkung hervor, die zwischen 262 und 263 am rande mit roter tinte eingetragen ist, und lautet: 
The day of dome shuld come in here;

Uor pe defawte of pe wrytere

At pe XIII leef afore hyt ys;

Seche hyt pere, pou shalt nott mys.

Diese zeilen lehren uns also, dass mindestens 12 blätter (etwa 2160 zeilen) am anfange fehlen, auf denen die ersten neun gedichte und der anfang des zehnten standen, in dessen mitte die jetzt vorhandene handschrift beginnt.

Die erste zeile des ersten vorhandenen blattes ist die zweite einer strofe von dreizehn zeilen - in solchen ist das erste gedicht abgefasst - , in der der dichter vor den todsunden warnt, die schreckliche strafen tuber die menschen bringen. Die folgende strofe (13-25) bringt dann den gegensatz im lobe des gerechten. Sodomie, totschlag, erpressung, erbschleicherei, mord, diebstahl und ehebruch - „des teufels werke" - werden dann gegeisselt, besonders aber die drei letzten:

48 Fore morper Cayme cursud of God was he, $\&$ fore peft penys al day hongud pay be Fore avoutre vengans had kyng Dave, Fore brekyng of pe sacrement.

Dann wendet sich der dichter an priester und pfarrer: Sie vor allen anderen wurden ewig bestraft werden, wenn sie solche stunden begingen und dadurch anderen ein böses beispiel gäben; denn sie wären ja grade erwählt, keusch zu leben, ihren heiligen stand auch heilig zu halten in vollkommener liebe und in wohlthätigkeit, und die ubelthäter zu bessern. Mit der sechsten strofe (65) richtet er sich dann an die eheleute; sie sollen in der ehe nach dem gesetze leben, das sei gott wohlgefällig, der ja selbst von einer jungfrau geboren sei; dann wendet er sich gegen die geldheiraten (!!); die ehe solle nur auf reiner liebe beruhen, so machten es auch alle tiere, nur der mensch allein stundige, wenn er in wilder ehe lebe und ehebruch treibe. „Ich aber weiss ein mittel:" fährt Audelay fort, „Verlasset die stunde, seid freundlich gegen gott und die menschen - kein anderer weg fuhrt zum himmel - und thuet rechtzeitig busse; denn gott hat gesagt: Nolo mortem peccatoris. - Was das sakrament gebunden hat, kann der menseh nicht lösen (130-142). Liebe gott uber alles and deinen nächsten wie dich selbst; schwöre keinen meineid, halte den sonntag in ehren, liebe vater und mutter, erschlage keinen menschen, verleumde niemand, gieb Anglia. N. F. VI. 
kein falsches zeugnis, betrüge und stiehl nicht, schwöre die geilheit ab. Haltet diese gebote und denket ans ende (143-159). - Noch ein andres heilmittel giebts, das sind die sieben gnadenwerke. Wer sie gethan hat, der wird bestehen am jungsten tage, wenn die ganze welt in flammen steht. Diese werke will ich euch nennen. Gebt den hungrigen speise, den durstigen trank; kleidet die nackten; besucht die kranken im gefängnis (nicht etwa: die kranken und gefangenen); begrabet die toten; beherberget die armen wanderer, und lehret die unklugen. Besonders verdienstlich aber ist die mildthätigkeit gegen die armen. - Auch deine funf sinne musst du wohl kennen, und wissen, dass du vor den tieren voraus hast, dass da gutes und böses unterscheiden kannst. Du hast die freie wahl, in den himmel oder in die hölle zu kommen; also verlasse den 'feind' und denke immer an Christi leiden (186-210). Glaube, hoffnung und liebe mögen den grund deines lebens bilden. Wenn dn deinen nächsten liebst, so liebst du auch den herrn. Barmherzigkeit ist die hauptsache, denn sie lehrt jeden das zu thun, was er an sich gethan zu sehen wunschte. So wird gott dir gnade gewähren am jungsten tage, wenn du mit den armen das deinige geteilt hast, denn die armen werden dann die richter sein uber die reichen (250-262).

Hier folgt dann jene anmerkung, nach welcher eigentlich ein gedicht uber das jungste gericht hier hätte folgen sollen, und daran schliesst sich unmittelbar ohne besondere uberschrift das zweite gedicht.

\section{Zweites gedicht (263-1275).}

Dieses zweite, das - wie schon erwähnt - in der handschrift als elftes bezeichnet wird, ist das längste gedicht der ganzen sammlung; es ist in derselben strofenform geschrieben wie das erste. Fast jede der dreizehnzeiligen strofen hat eine lateinische uberschrift, in der regel eine bibelstelle. Die erste lautet: "De concordia inter rectores fratres et rectores ecclesiae"; hier heisst es wieder: „Keiner kann erlöst werden, der nicht glauben, hoffnung und liebe hat." Dann „Hæc est fides catholyca": Schon bei der taufe haben wir gott gelobt, die teufelswerke zu meiden und gottes gebote zu halten; dies gelubde durfen wir nicht brechen. - "Time dominum et mandata eius serva": Wer dies gelöbnis bricht, wird keine gnade 
finden, wenn er nicht vorher auf erden busse thut. - „Sapientia huius mundi, stultitia est aput dominum": Der hat wahre weisheit, wer ein reines leben fubrt. - So geht es dann weiter; fas $t$ jede strofe trägt - wie gesagt - eine thberschrift, die aber nicht immer auf den inhalt der strofe bezug hat.

Zunächst wird namentlich vor den heuchlern gewarnt, die viel von ihrer liebe zu gott reden, aber nicht darnach thun. Merkwürdig ist die erwähnung von Salomon und Markolf in der 'Vox populi vox dei' tberschriebenen, aber kaum erklärbaren strofe $328-340$ :

328 I Marcol pe more fole mon on my mad wyse, I send pe broder Salamon to say as I here,

330 Hou homle hosbondusmen here hertis pai aryse;

pai woldon pai wrozton wysely pat schuld ham lede \& lere.

Do pi message mekely to pryst \& to frere, pai are pe lanternys of lyf, pe lend men to lyzt,

Bot pai be cazt with couetyse, with consians vnclere, Azeyns pe lauys of here lord, reson \& ryzt.

Hit is nozt vnknow

Comawnd hem in al wyse

Neuer on oper pai [dur] dyspyse

Fore here cursid couetyse

340

Here horne is eblaw.

$\mathrm{Zu}$ 'Marcol pe more fole' vergleiche man, was Kemble in seiner ausgabe von 'Salomon and Saturn' tuber die französische fassung der sage mitteilt: „The popular feeling took another turn with Marcol, and dubbed him deservedly 'Marcol le foole' " (s. 76), und: „Ms. Trin. Coll. Cam. R. 3.19. This was written in England .... and has the following epigraph: 'Veez cy une desputacoun entre Salamon ly saage, \& Marcoulf le foole', and in addition the following running title: 'Salamon pe wyse, and Marcolf pe more foole'." Auf 'Marcol pe more fole' beruft sich Audelay später noch einmal in der vorletzten (vorhandenen) strofe dieses gedichtes; ich will anch diese ganz hierher setzen :

Cuius finis bonum (!) ipsum totum bonum.

1250 pus Salamon hap sayd pe sop verement,

As Marcol pe more fole warned hym - I wene -;

Bot zif his drazt be draun wel pai goun (= go \& ?) wil be schent

$\&$ schal turne treule to turment \& to tene.

Haue mynd on pis mater, ze wot what I mene,

Bluster not furp vnblest as Bayard pe blynd, 
Bot cal azayne charyte with consians clene, \& wry not fro godis word as pe wrop wynd,

Herkyns hit as pe hynd;

1260

Apon zour leuyng take good eme, \& bep seche as ze schul seme,

Fore be zour dedis men wyl zou deme.

Here I make an end.

Von 'Bayard pe blynd' heisst es in der dritten strofe vorher:

1214 Bot al blustyrne furp vnblest as Bayard pe blynd;

und vorher schon in der vierten strofe, die auf jene erste folgt, in der von Markolf die rede ist:

384 Make moche of good mon, on hym take good hede,

Loke ze bete not Bayard for bryd ne fore brend.

„Schnöden gewinn - so heisst es in den vorhergehenden strofen $(341-379)$ - und die eitelkeiten der welt sollst du meiden und die priester nicht verachten"; und dann später : „Wenn ein armer priester recht fromm ist und viel betet, so wird er leicht verleumdet und 'pay lekon hym to a lollere \& to an epocryte' (395); die leute halten nicht viel von solchen frommen priestern, und daher giebt es derer denn auch nur noch wenige."

Dann folgt eine ganze strofe (407-419) uber einen klostergenossen des dichters, den dieser mit bitterem hohne schildert. Die tberschrift lautet: "Increpasti superbos; maledicti qui declinat amantis ( $!=$ a mandatis tuis. Ps. 118, 21). Dann heisst es: „Unser freund Johannes ist ein fröhlicher mann in gesellschaft; er spielt die harfe und versteht zu singen, und er giebt viel geld aus. Es ist doch schade drum, dass er kein pfarrer oder vikar ist. Er kleidet sich stets sehr schön, sein gurtel ist mit silber verbrämt, und ein dolch hängt daran. Jedermann gefällt seine börse, sowohl den mädchen als den weibern. Wahrlich! Er soll nicht von uns ziehen; denn dann wäre es um unsere lust gethan, da er uns alle fröhlich machen kann. Gott möge ihm ein langes leben schenken!"

Hieranf folgt ein inbrtunstiges gebet fur alle die, die sich so gegen gott versundigen (420-428). Am schlusse dieser strofe heisst es: "Hier sollt ihr nun hören von geistlichen männern, was für ein leben die fuhrten, um gottes geheiss zu erfullen." So schliessen sich denn zunächst die gebote des St. Benet uber das klostorleben an, die dem hörer in ausfuhr- 
licher breite ans herz gelegt werden (432 ff.). Namentlich verweilt Audelay lange beim bibellesen, von dem er sagt: 'Alle gottesgelehrten befahlen in Christi namen, an der bibel nicht zu mäkeln, da sie gottes eigentum ist." - Dann folgt ein lob der woblthäter der kirehe, für die auch eifrig gebetet werden solle.

Dann wendet er sich an die grossen des landes: Sie sollen sich liebe zu erwerben suchen, sie sollen auch die priester zu rate ziehen, damit die sünde nicht das land zerstöre; an den frommen vorfahren sollen sie sich ein beispiel nehmen. Wieder schärft er dann Christi ausspruch ein: Diligatis invicem.

In der folgenden strofe ( $536 \mathrm{ff}$.) erinnert er wieder an die vergänglichkeit der welt und sagt da:

542 Fore all pe worchyp of pis word hit wyl wype sone away

Hit fallus \& fadys forp, so dop a chere fayre.

Ich fasste dies anfänglich so auf: 'Fällt und welkt wie ein schönes gesicht'. Später sah ich in Halliwells ausgabe, dass dieser liest: '. . wie ein kirschenjahrmarkt', der als sinnbild der küze des menschlichen lebens und der vergänglichkeit aller freuden gebrancht wird. Ich glanbte zuerst, diese erklärung sei bei den haaren herbeigezogen, und die meinige sei besser; nachher aber ersah ich aus Murray's New English Dictionary, dass nicht allein cherry-fair noch viermal in dieser bedeutung belegt ist, sondern auch einmal cherry-feast. $\mathrm{Ob}$ aber nicht dennoch wegen des zeitwortes fadys an unserer stelle meiner auffassung der vorzug zu geben ist, wage ich noch nicht $\mathrm{zu}$ entscheiden.

In der nächsten strofe (549 ff.) wird das beispiel des reichen mannes und des armen Lazarus verwendet, um vor ungehorsam gegen gottes gesetze zu warnen; dann (562 ff.) wird demut empfohlen, hochmut sei es ja gewesen, was den Lucifer zu falle und in die hölle gebracht habe.

In solcher weise geht es nun in diesem gedichte weiter; er klagt darüber, dass die laien in die kirchlichen wahlverhältnisse eingreifen (601 ff.), er wettert gegen die schlemmer und empfiehlt immer wieder die liebe gegen gott und den nächsten als das beste mittel die ewige gnade zu erlangen.

Die strofe $654 \mathrm{ff}$. beginnt:

My blessid broder Salamon, spesialy I pe pray,

Meue pis mater maysterfully to prest $\&$ to frere, 
und die zweite folgende so:

680 I say pe, broder Salamon, tel in pi talkyng

Furst of pe frerys; .....

Audelay lobt dann die bettelmönche; reden sei besser denn schweigen, wenn man mangel habe. Dann aber wendet er sich gegen die, die zu viel geld für messen einnehmen; er vergleicht sie mit Judas, der den herrn für geld verraten habe; es sei gegen gottes gesetz, mehr zu verlangen als man zum unterhalt nötig habe (742).

Dann redet er die brtider selbst an: Sie sollten nicht den armen das geld wegnehmen, sondern nur bei den reichen betteln, aber allen gleich predigen; „denn ihr findet eine menge, die viel ärmer sind als ihr selbst; denen sollt $\mathrm{ihr}$ wieder mit euren mitteln helfen" (-783). Namentlich schärft er ihnen dann noch ein, dass sie auch so leben sollen wie sie predigen:

793 A prechur schuld lyue parfytly

\& do as he techys truly,

Ellis hit is ypocresy.

Wieder wendet er dann das beispiel vom reichen manne und von Lazarus an.

Des weiteren kommt einmal eine strofe (810 ff.) uber die priester, die zu leichtsinnig leben, und namentlich nach der beichte wieder drauf los stindigen: dann eine solche (823 ff.), in der arme leute gewarnt werden, ihre söhne nach Oxford 'to scole' zu schicken, da solche einst nur durch Simonie zu stellen kommen könnten.

"In drei teile sollte das vermögen der kirche geteilt werden; ein teil diene zum unterhalt der priester, ein zweiter dazu, das ansehen der kirche aufrecht zu erhalten, der dritte werde unter die verarmten gemeindemitglieder verteilt" (849 ff.).

Von zeile 939 ab folgt ein langer ausfall auf die Lollarden, für die man nicht einmal beten solle! Selbst seinen bischof greift Audelay an (977 ff.), weil er die leute stundigen lasse, und zum dank dafür geld von ihnen nehme.

Gegen solche habgier der geistlichkeit eifert er dann weiter (1016 ff.), 'A foule defaute' sei es, wenn ein priester zweimal am tage eine messe singe nur um schnöden mammons willen; wurde er es um Christi willen thun sollen, so wurde er sicher murren; so aber verrät er den heiland grade wie Judas. - Sehr ernstlich mahnt er auch, dass jeder mindestens 
einmal jährlich beichten mulisse (1081 ff.). - Weiter heisst es (1094 ff.), dass, wenn ein priester unwtirdig seine messe singe, das sakrament dadurch zwar nicht verdorben werde, der priester aber eine todsunde begehe. "Obgleich nur e in gott im sakramente auf dem altare geopfert wird (1146 ff.), so erhält doch jeder anwesende seinen anteil, und wenn er wahre reue zeigt, wird er auch in gnaden angenommen."

Ein hubsches bild wendet Audelay vers $1159 \mathrm{ff}$. an : ${ }_{\text {nJede }}$ todsunde ist eine wunde, die der 'feind' dem menschen beibringt; wenn euch daher der feind besiegt hat, so sollt ihr wollt ihr wieder gesund werden - zum arzte gehen, der die wunde untersuchen und heilen kann; beichte und reue sollen die salbe sein. Dann kannst du wieder mit dem feinde fechten und ihn besiegen." - Die busse aher, die die priester anflegen, solle nicht zu schwer sein; auch Christus habe nur gesagt: 'Vade in pace, amplius noly peccare' (1192).

Wohl den abschluss dieses langen gedichtes, das sich hauptsächlich an die priester wendet, haben wir $1263 \mathrm{ff}$. in einer strofe, in der sich Audelay gleichsam entschuldigt, dass er so offen die wahrheit gesagt habe uber kirchliche missbräuche; er sei durchaùs nicht parteiisch; er könne nicht lìgen und nicht schmeicheln und wolle gott nicht erztirnen; er könne nicht anders.

Hinter dieser strofe, mit der das 7. blatt der handschrift abschliesst, ist offenbar wieder eine grössere lücke; denn während das bisherige lange gedicht, wie wir sahen, die nummer 11 trägt, ist das nächste, dessen anfang wir haben (in der zweiten spalte der vorderseite des 8 . blattes), mit 16 bezeichnet, sodass das ende von 11 (?), die nummern 12,13 und 14 und der anfang von 15 fehlen. In der mitte dieses 15 . gedichtes beginnt blatt 8 .

\section{Drittes gedicht (1276-1377).}

Das achte blatt beginnt mit der dritten zeile einer dreizehnzeiligen strofe (mit derselben form wie die der ersten beiden gedichte), in der davor gewarnt wird, böses über seinen nächsten zu sagen.

Die folgende strofe beginnt:

1287 pe VII. vertu ys good conselyng,

so dass in dem fehlenden anfang die sechs ersten tugenden 
besprochen sein mtissen; in der nächsten ( $1300 \mathrm{ff}$.) wird die achte tugend, 'das heilige gebet', behandelt, und in der folgenden die neunte, die darin besteht, gott tuber alle dinge zu lieben.

Das gedicht endet mit zwei strofen (1352 ff.), die im wesentlichen den inhalt der strofe (4337 ff.) 'Meruel ze not of pis makyng' haben, die oben (s. 179) abgedruckt ist; die letzten zeilen lauten hier:

$1374 \mathrm{Y}$ made pis wit good entent,

In hope pe raper ze wolde repent,

Preyes for me pat bep present,

My name hyt ys pe blynde Awdelay.

Bis hierher ist - wie schon bemerkt - alles in einer dreizehnzeiligen strofe abgefasst. Die reimstellung ist durchweg so wie in den hier mitgeteilten proben, also: $\mathbf{a} \mathbf{b} \mathbf{a} \mathbf{b}$ b c b c d e e e d, z. b. heuen : pouerte : euen : body : be : passe : pe : classe : ay : kyng : lykyng : endyng : day. Nach Schipper (Metrik I, 395 und 423) scheint die strofe in dieser art im mittelenglischen nicht weiter vorzukommen; tubrigens ist diese strofenart tuberhaupt die einzige, die Schipper von Audelay belegt. Im ersten und im dritten gedichte sind die einzelen verse meist viertreffig, die senkungen werden nicht grade regelmässig behandelt, und es giebt eine sehr grosse anzahl von ungemein holperigen versen. Zuweilen haben die erste und die letzte zeile des zweiten teiles (d) nur zwei treffe; auch solche mit dreien kommen vor. Dies gilt auch für das zweite gedicht, in dem aber nur die 10., 11. und 12. zeile viertreffig sind, während der erste teil der strofe allitterierende langzeilen mit sieben treffen aufweist, doch ist naturlich auch hier keine regelmässigkeit mehr vorhanden; oft sind nur zwei, oft vier und mehr stäbe in einer zeile.

\section{Viertes gedicht (1378-1516).}

Das vierte gedicht (nach der handschrift nr. 16) trägt die uberschrift 'De effusione sanguinis Christi in remissionem peccatorum' und besteht aus 23 sechszeiligen strofen; die verse sind viertreffig, nur in der dritten und sechsten zeile zuweilen dreitreffig. Reimstellung: $\mathbf{a} \mathbf{a}$ b c c b. Auch von der allitteration wird hie und da gebrauch gemacht. - Die ersten zeilen lauten: 
1378 An holy prayer here bygynnes In remedy of seue[n] dedly synnes. VII blodes Crist he bled.

Das gebet behandelt das siebenfache blut Christi. Die ersten drei strofen bilden die einleitung; dann beginnt das gebet mit der vierten; diese und die folgenden vierzehn beginnen alle mit ' $O$ Jesu'; die ersten sieben behandeln je ein blut, z. b. heisst es in der ersten: ' $O$ Jesu, wegen des blutes, das du bei deiner beschneidung vergossen hast, vergieb mir meine fleischlichen sünden' usw. Dann werden zum ausgangspunkte der einzelnen strofen gemacht: Der pfahl, an den Jesus bei der geisselung gebunden wurde (1438); das schweisstuch der Veronika; das kreuz; die drei nägel; das gefäss für essig und galle; die lanze; die 'louely' leiter, die zange und der hammer bei der abnahme vom kreuze; schliesslich auferstehung und himmelfahrt.

'Funfzehn vaterunser', heisst es schliesslich (1487), 'bete ich für dein heilig leiden, und funfzehn Ave an die jungfran Maria, dass sie für mich bitten möge.' Dann folgt die aufforderung, jeder solle dies gebet täglich beten; segen aber verdiene sich anch der, der es einen andern lehre.

\section{Funftes gedicht (1517-1558).}

Das fünfte (17.) gedicht trägt die thberschrift: 'Quomodo Jesus fuit reprobatus a Judeis' und ist in demselben versmasse und in derselben strofenform geschrieben wie das vierte. Es ist wieder ein gebet. In den ersten vier strofen werden alle leiden Christi aufgezählt; dann heisst es: 'Bei diesen leiden bitte ich dich: Errette mich von den höllenstrafen.' Die letzte der sieben strofen empfiehlt auch dies gebet wieder den gläubigen zu eifriger benutzung.

\section{Sechstes gedicht (1559-1620).}

Dieses sechste (18.) gedicht ist halb lateinisch und halb englisch. Die tiberschrift lautet: 'De Psaltero passionis'. Es beginnt so:

1559 Wele is him pat wele can

Sai pe sauter of pe passion;

Here pus pou schalt begyn

In pe remission of pi synne. 
Dann folgen zwölf zeilen Latein, deren letzte mit den englischen worten 'per charite' endet. Dann geht es weiter:

1575 Instid of pi paternoster pis pou take $\&$ pis [si?] pi aue fore Cristis sake.

Wieder vier zeilen Latein; dann:

1581 \& say on pi bedis in pis manere

As pou didist our lade santere.

When pe sauter hit is edone,

pen say pi crede with hit anon;

pen in pe worchip of Cristis passion

Say pis hole oresoune.

Dann folgt dies gebet (lateinisch) in 34 zeilen, das sich dem inhalte nach fast wörtlich deckt mit dem englischen in den zeilen $1517 \mathrm{ff}$. (s. das vorige gedicht).

Siebentes gedicht (1621-1734).

Das siebente (19.) gedicht heisst: „De septem verbis Jesu Christi pendentis in cruce". Die strofe ist dieselbe wie die des vierten and des funften gedichtes; ihre zahl ist 19. Die erste und die 3. -15 . beginnen auch hier mit: 'O Jesu'. - Der inhalt dieses gebetes is kurz: 'Vergieb mir, Jesus, kraft deiner sieben worte am kreuze das, was ich hier von den sieben todsunden begangen habe. Da sprachst: 'Vergieb ihnen, denn sie wissen nicht, was sie thun'; so vergieb du nun meinen feinden. Du sprachst: 'Morgen sollst du mit mir im paradiese sein'; so führe mich auch dahin. Du empfahlst deine mutter dem Johannes; um ihrer liebe willen zu dir habe gnade mit meiner seele. - In dieser weise geht es weiter. Auch hier ist der schluss eine mahnung, das gebet häufig zu sprechen; volle vergebung der sunden werde der lohn sein.

\section{Achtes gedicht (1735-1826).}

Das achte (20.) gedicht handelt: 'De salutatione corporis Christi Jesu'. Es wird eingeleitet durch eine sechszeilige strofe, wie sie die vorigen gedichte aufweisen; die dritte und die sechste zeile sind dreitreffig. Inhalt: Wenn du das sakrament siehst, grtisse es mit folgenden worten. - Das gedicht selbst hat elf strofen. Die ersten acht sind achtzeilig mit der reimstellung a a a b cceb; die vierte und die achte zeile haben drei treffe, die tubrigen aber sind meist stabzeilen in den ersten 
sieben, sonst viertreffige, und $\mathrm{nur}$ viertreffige in der letzten dieser acht strofen. Die letzten drei strofen sind nur sechszeilig wie die frtheren, aber sehr unregelmässig.

Fast jede zeile dieses gedichtes beginnt mit dem ausrufe: Hayle! - 'Deine gottheit kann mit fleischlichem auge nicht gesehen werden; ich glaube, dass du wahrhaft in diesem brote bist; ich glaube an deine empfängnis, an deine geburt usw. Gewähre mir gnade, dass ich deinen geheiligten leib empfange.'

Die letzte der ersten acht strofen verheisst wieder eine vom Papst 'Enocent' geschaffene vergebung der stunden fur den, der dies gebet sagt.

Die drei ubrigen strofen bringen eine kurze wiederholung des inhaltes der vorhergehenden. Dann folgt noch ein lateinisches gebet.

Neuntes gedicht (1827-2240).

Das neunte (21.) gedicht handelt 'De meritis missæ, quomodo debemus audire missam'; es ist bedentend länger als die letzten vorhergehenden und besteht wieder aus jenen sechszeiligen strofen, in denen hie und da der stabreim verwendet ist.

Der dichter sagt am anfange, er wolle davon reden, wie man die messe hören mulsse. 'Wenn du nach der beichte zur messe gehst, so bertihrt ein engel dein angesicht, dass du den leib des herren sehen kannst. Wer das sakrament würdig empfängt am tage seines todes, der wird dadurch erlöst; ist er aber in todesstinde, so ist es sein verderben; beweis: Judas. - Wer sein vaterunser, sein ave und sein glaubensbekenntnis nicht kann, der kann auch nicht erlöst werden; denn in dem vaterunser ist in den sieben bitten alles enthalten, was du und dein nachbar bedurft; funf verehrungen enthält das ave, zwölf sätze das glaubensbekenntnis. Aber auch die zehn gebote musst du kennen und halten, und wissen musst du, welches die sieben todstinden sind. Hast du dieser eine auf dem gewissen, so sollst du sie beichten. Dann magst du in der kirche niederknieen und beten, zunächst für dich selbst, dann für deine eltern und dann für deine tibrigen angehörigen, für deine freunde und für deine feinde, für deine wohlthäter, für den priester, der die messe liest, und für alle, die in der kirche sind (1994). Sankt Bernhard und Sankt Beda sagen beide, dass die messe 
niemand verbessern noch verschlechtern könne; auch könne niemand all ihr lob völlig sagen.

Wenn ihr auch nicht alle laut nennt, fur die ihr beten wollt, so könnt ihr sie doch im sinne behalten. Jeder kann so an der messe teil nehmen, nur nicht diejenigen, die fur ewig in der hölle schmachten.

An jedem tage sollst du eine messe hören; wenn es dir nicht möglich ist, so sollst du wenigstens zu hause beten, während in der kirche die messe gelesen wird, und du wirst ihrer segnungen auch so teilhaftig werden. Wenn du am morgen keine messe hören kannst,

2083 Loke ze do be vndorne

Or ellis be mydday.

Zu 'vndorne' (= vormittags) vgl. Stratmann-Bradley bei undern. Ich hatte zuerst vndorue gelesen, verfuhrt durch das reimwort morwe in 2082; dieses ist aber zu morne zu ändern.

'Augustin hat es besonders betont, dass man von dem angenblicke an, wo der priester das messgewand anzieht, nicht sprechen dürfe, denn sonst würden die bösen geister alle eure worte aufschreiben.'

Es folgt nun eine merkwürdige geschichte ther den heiligen Augustin, die kurz folgenden inhalt hat: „Ehe Augustin nach England kam, wohnte er in Rom mit dem heiligen Gregor zusammen. Eines tages wollte dieser eine messe lesen und bestellte den Augustin als leser des evangeliums. Als dieser nun las, sah er drei weiber da sitzen, die sich geschichten erzählten. Er verstand alles. Auch sah er durch ein fenster einen sitzenden tenfel mit feder, tinte und pergament. Der schrieb so lange, bis ihm das pergament ausging. Er begann, es mit den zähnen auseinander zu ziehen, und Robin (?) half ihm; dieser that das so stark, dass er mit dem kinnbacken gegen einen marmorstein fiel, was einen schall verursachte, dass alle anwesenden erschraken. Augustin lachte. Gregor aber schilt ihn nach der messe in milder weise; da erzählt ihm Augustin, was er gesehen und gehört habe, und fuhrt ihn zu dem fenster, wo sie nun zu ibrem entsetzen schwarze blutspuren auf der fensterbank bemerken. Gregor befahl darauf, dass diese geschichte bei jeder messe erwähnt werde."

'Seid also stille, wenn ihr in der kirche seid!' 
Nun wiederholt sich der dichter, eine ganze strofe ans dem anfange des gedichtes kehrt wieder (2187): 'Bete für dich und die deinen' usw.; dann aber geht es anders weiter: 'Bete für das wetter und fur den frieden, fur alle christenheit. Dann sage drei vaterunser in Christi namen, dann noch fünf, um deine fünf sinne so gebrauchen zu lernen, dass du erlöset wirst, dann noch fünf an den heiligen geist und zehn fur die ubertretung der zehn gebote.'

Dann kommen auch wieder wiederholungen: 'Höre die messe usw.; die kraft ihres segens kommt daher, dass Christus in der whiste das brot and die fische segnete bei der speisung der 5000 mann. - Allen, die 'pis sermon' gehört haben, gewährt der heilige Gregor 100 tage ablass. Jesu möge euch erlösen!' So endet dieses tuber 400 zeilen lange gedicht.

\section{Zehntes gedicht (2241-2312).}

Das zehnte (22.) gedicht ist uberschrieben: „De confes sione generali "; es wird eingeleitet durch 22 zeilen (elf reimpaare) mit der besonderen tuberschrift: 'Quomodo dominus Jesus apparuit Sancto Gregorio': Dem heiligen Gregor sei einst bei der messe der herr erschienen; jener habe in folge dessen einen ablass gestiftet; wer den erwerben wolle, solle die folgende beichte sagen.

Diese selbst besteht aus 20 reimpaaren von viertaktigen versen; das schlusspaar lautet in tibersetzung: 'Siehe zu, dass du dieses gebet ebrerbietig hersagst'.

Es schliessen sich dann noch fünf reimpaare an, die wieder eine besondere tuberschrift tragen, und zwar: Quomodo Jesus in cruce rogabat patrem pro inimicis: „Jesus, du batest, dass gott deinen feinden vergeben möge; vergieb du deshalb meinen feinden und mir."

Es folgen zwei leere zeilen, in denen etwas ausradiert zu sein scheint; doch fehlt wohl nichts.

Elftes gedicht (2313-2719).

Das elfte (23.) gedicht bandelt: „De visitatione infirmorum et consolatione miserorum"; es besteht aus 31 dreizehnzeiligen strofen im versmasse des ersten und des dritten gedichtes. 
Es beginnt so: „Saynt Ancelyne (Anselm) machte diese abhandlung ('tretys'), wie ihr auf gott eure hoffnung setzen sollt und ihm alle eure stinden beichten, wenn es zum sterben geht. Wer aber seine studen nicht schon vorher bekennt, der wird gar sehr bedruckt sein, wenn er erkrankt aus furcht vor dem tode. - Um Christi willen gebt almosen (2391); wenn du im leben einem armen auch nur einen pfennig oder speise und trank oder kleidung oder herberge giebst, so thust du gott einen grösseren wohlgefallen, als wenn du tausend berge goldes zu geld machen liessest, um es nach deinem tode austeilen zu lassen. So ist auch mehr wert eine messe hier in deinem leben als tausend nach deinem tode (2404). Gregor sagt: 'Löschet das höllenfeuer schon hier'; Anselm: 'Welche heimsuchung gott dir auch schicken mag, immer musst du zufrieden sein'.

Denkt an Maria Magdalena (2456), an Petrus, an 'Tohmas of Ynd'. So werden alle begnadigt werden, die hier busse thun, wenn es noch zeit ist; denn ther einen suinder, der busse thut, ist mehr freude im himmel usw. Aber alle, die nicht an den herrn glauben, werden von ihren sünden verklagt werden am jungsten tage. Seid ihr christen genannt und lebt nicht christlich, so tragt ihr den namen vergebens und werdet in schande fallen. Christus aber, der fur dich gelitten hat, hat eine salbe fur jede wunde, nämlich die sieben sakramente. Wenn du wahre reue hast und das abendmahl im glauben empfängst, so wirst $d u$ am jungsten tage bestehen; nicht aber die ketzer und renegaten, für die giebt es keine erlösung."

Immer wiederholt sich der dichter and wird dadurch ungeheuer eintönig; so wiederholt er hier wieder das ganze glaubensbekenntnis (2612) und die mahnung zur beichte. Dann heisst es weiter (2651): 'Mlachst du dein testament, so bezahle zuerst deine schulden.'

Am schlusse des gedichtes steht wieder die uns schon von zwei anderen stellen her bekannte strofe 'Meruel ze not of pis makyng'; diesmal aber lauten die dritte und die vierte zeile so:

2692 pis was seynt Ancelme cownselyng, pat was a bischop ful holy;

und der zweite teil: 
2698 3e curatis, here I zou pray,

pat han mon soule in zour kepyng,

Let red pis trete fore one pyng

To pe seke at here endyng;

pus counsels zou pe blynd Audley.

Nach der letzten strofe, in der der dichter nochmals zur busse mahnt, folgt die lateinische schlussbezeichnung 'Explicit visitacio infirmorum \& consolacio miserorum', und dann folgen diese vier zeilen:

2716 I pray zou, serys, per charyte, When ze han red pis trenly, pen redis pis passion

2719 What Cryst sofyrd fore synful men.

'pis passion' folgt aber nicht unmittelbar, sondern erst beim 2793. verse. Dazwischen steht ein anderes gedicht.

\section{Zwölftes gedicht (2720-2792).}

Dieses zwölfte gedicht schliesst sich ohne uberschrift an 2719 an, trägt auch keine besondere nummer, und besteht ausser der anfangsstrofe aus elf sechszeiligen strofen mit der reimstellung $\mathrm{a} a \mathrm{~b} \mathrm{ccb}$, die aber sogar meist $=\mathrm{a} \mathbf{a b}$ a a b wird; die verse sind viertreffig, die dritten und die sechsten aber nur zweitreffig.

Die erste strofe, die gleichsam die uberschrift bildet, lautet:

2720 Here schul ze here a treu lessoun,

Hou fayp \& charyte away is gon.

$\left.\begin{array}{l}\text { Multis diebus iam peractis } \\ \text { Nulla fides est in pactis }\end{array}\right\}$ Videte!

$\left.\begin{array}{l}\text { Mel in ore, verbis lactis, } \\ \text { Fel in corde, fraus in factis }\end{array}\right\}$

Cauete.

Das gedicht selbst behandelt die schlechtigkeit der welt, indem es diese lateinischen sätze ubersetzt und weiter ausfuhrt. - 'Beichtet daher, ich rate euch gut, ich der blinde Audelay.'

Dreizehntes gedicht (2793-2916).

Das dreizehnte gedicht ist in der handschrift mit dem vierzehnten unter einer nummer (24.) vereinigt und unter der uberschrift: „De passione domini nostri Jesu Christi \& de horis canonicis."

Die 'passion' besteht aus 15 achtzeiligen strofen mit der reimstellung $\mathrm{a} b \mathrm{a} \mathrm{b} \mathrm{b} \mathrm{c} \mathrm{b} \mathrm{c}$; die zeilen sind viertaktig. 
Sie beginnt 80: 'Johann XII. machte dieses evangelinm und verhiess hundert tage ablass dem, der es drei tage vor seinem tode betet.' Dann werden die leiden des herrn geschildert. Die ersten fünf strofen endigen mit der zeile:

$\&$ al hit was fore loue of pe.

Gegen schluss des gedichtes (2873) beisst es: „Das litt der herr für dich; wie willst du es ihm vergelten? Was littest du für ihn? - Sage daher dies gebet." Dieses gebet umfasst die letzten vier strofen, von denen die vierte vier zeilen mehr hat (mit der reimstellung a a b b), die lauten;

2913 Amen, Jesu, now I pe pray, Haue mynd \& merce on blynd Audlay pat mad in englesche pis passion Fore synful men to haue mynd peron.

Vierzehntes gedicht (2917-3006).

Beim 2917. verse beginnt, unter der besonderen uberschrift 'Hic incipiunt horæ canonicæ passionis Jesu Christi', das vierzehnte gedicht, das aus zehn neunzeiligen strofen besteht, die die reimstellung $\mathrm{a}$ a $\mathrm{a}$ a $\mathrm{b}$ e c c b haben; die zeilen mit $a$ sind stabreimende langzeilen, die mit $b$ drei- oder viertreffig, die mit c viertreffig; auch diese letzten haben viele stäbe.

Das gedicht beginnt mit einer anrufung Christi. Dann heisst es: 'Nun sollt ihr hören, welch hartes leiden Christus durchzumachen hatte.'

Von den ubrigen neun strofen hat jede eine besondere uberschrift. Diese lautet bei der ersten: 'Patris sapientia veritas divina'; inhalt: 'In der morgenstunde wurde Christus gefangen genommen.' Ueber der zweiten steht: 'Hora prima ductus est Jesus ad Pilatum'; uber der dritten: 'Crucifige! clamatant hora tertiarum'; in ihr kommt wieder die bezeichnung des vormittages, at vndor (!), vor; tuber der vierten steht: 'Hora sexta est cruce conclauatus'; uber der funften: 'Hora nona Dominus Jesus expiravit'; uber der sechsten: 'De cruce deponitur hora vespertina'; uber der siebenten: 'Hora completore datur sepulture'. Jedes dieser ereignisse wird in der strofe selbst ausfuhrlich beschrieben.

In den beiden schlussstrofen folgt wieder eine anrufung Christi und das versprechen vollkommenen ablasses fur den, der dies mit ehrfurcht betet. 
Funfuehntes gedicht (3007-3214).

Das funfzehnte (25.) gedicht trägt eine lateinische uberschrift von funf zeilen und behandelt einen brief, den Christus mit eigner hand geschrieben haben soll an Petrus in Gazon uber die heiligung des sonntages. Hier finden wir wieder die dreizehnzeilige strofe, in deren zeilen wieder oft, aber nur gelegentlich, der stabreim verwendet ist.

Die ersten strofen enthalten eine verfluchung derjenigen, die den sonntag nicht heilig halten. 'Man soll zur kirche gehen oder zur wallfahrt, die kranken besuchen und dem herren dienen. Man darf aber keine botschaft ausrichten, auch bart und haupthaar nicht scheeren lassen, keine kleider waschen, nicht brauen und nicht backen. - Der sopntag soll aber gefeiert werden von der none des samstags bis zur ersten stunde des montags'. - Dann geht es weiter (3111): 'Bezahlet nur ordentlich eure zehnten an meine priester, die tag and nacht für euch beten. - Die priester aber (3137) sollen diesen brief zum gegenstande ihrer predigt machen, sonst werden sie ewige strafen empfangen.'

Dann heisst es weiter (3150): „Christus sandte diesen brief an Petrus nach Gazon, der dort zuerst bischof war. - Der sonntag ist heilig, an ihm machte gott die neun engelchöre, an ihm blieb Noahs arche auf dem 'hil of Armony' hangen. Feiert den tag daher, Christus selbst hat es ja besonders befohlen in diesem briefe;

3174 Crist wrot hit with his fyngers alon

To warne his pepel, lest pay were chent.

Kein mensch kann die herrlichkeit beschreiben, zu der uns Christus einfuhren wird, wenn wir den sonntag heilig halten.'

Auch dieses gedicht schliesst wieder mit der bekannten strofe 'Meruel ze not of pis makyng'.

Sechszehntes gedicht (3215-3578).

Das sechszehnte (26.) gedicht ist auch wieder in der dreizehnzeiligen strofe (ohne stabreim) geschrieben; die lateinische uberschrift umfasst vier zeilen. Der inhalt ist, wie Michael den Paulus in die hölle fuhrte. Es ist - wie schon erwähnt - im Miscellany von Morris abgedruckt.

An das vorhergehende schliesst sich dieses mit der ersten zeile an: 
Das gedicht behandelt dann jene legende und schildert die höllenstrafen.

Als gegensatz zu dieser furchtbaren schilderung folgt gegen den schluss des gedichtes die erzählung, wie Paulus plötzlich eine schaar von engeln sieht, die eine fromme seele eben gen himmel tragen. - Dann werden sie von den höllenbewohnern angerufen, fur sie zu flehen; Paulus und alle engel thun das, und nun bewilligt der herr ihnen ruhe vom samstag abend bis zum montag morgen. - Als Paulus dann fragt, wie viele böllenstrafen es giebt, sagt Michael, sie seien unzählig. - 'Also richtet euch darnach, thuet rechtzeitig busse, damit ihr in den himmel kommt.'

Die vorletzte strofe (3553) ist eine fast wörtliche wiederholung der zweiten der handschrift (13-25), und die letzte ist wieder die bekannte 'Meruel ze not of pis makyng' mit geringen änderungen.

\section{Siebenzehntes gedicht (3579-3841).}

Das siebenzehnte (27.) gedicht ist das vorletzte der ersten abteilung und trägt die tiberschrift 'De misericordia Domini.' Es besteht aus achtzeiligen strofen von viertreffigen versen mit der reimstellung $a b a b b c b c$. Jede strofe schliesst hier mit dem lateinischen spruche 'Nolo mortem peccatoris'. Viele der strofen tragen eine besondere lateinische tuberschrift, mit einem bibelspruch. Das gedicht enthält eine mahnung Christi an gläubige und ungläubige, sein leiden zu bedenken. 'Vierzig tage habe ich gefastet, so sollt auch ihr vierzig tage fasten. Am aschermittwoch sollt ihr asche nehmen (3651), da ihr aus erde seid und wieder za asche werden sollt. Im fruhjahre sollt ihr beichten (3667). - Ihr, die ihr mich vergesset und mein leiden, euer will auch ich vergessen in euerer todesstunde (3707).'

Hinter der 3766. zeile, der letzten des 19. blattes, scheint wieder eine lücke zu sein, denn sie ist die vierte zeile einer strofe, während die erste des zwanzigsten blattes die zweite zeile einer strofe ist. Die strofenform ist dieselbe wie vorher; da auch der kehrreim derselbe ist, und dem inhalte nach das folgende zu dem vorhergehenden gehört, so wird hier vielleicht nur e in blatt ausgefallen sein.

Der inhalt ist: 'Teilet mit den armen.' 
In die strofe 3798-3809 sind funf lateinische zeilen eingeschoben. Dann heisst es: "Nehmet ein beispiel am heiligen Martin, der seinen mantel mit dem armen manne teilte, und denket an den reichen mann und Lazarus."

Der dichter schliesst dann mit einem lobe der heiligen dreieinigkeit.

Achtzehntes gedicht (3842-4349).

Unmittelbar an das letzte 'Nolo mortem peccatoris' des siebenzehnten gedichtes (3841) schliesst sich ein anderes ohne besondere nummer, ohne besondere uberschrift, uberhaupt ohne jedes trennungszeichen. Es besteht aus dreizehnzeiligen strof en mit viertreffigen zeilen von der bekannten form; ihrer sind 39. Die erste beginnt so:

3842 'Here I conclud al my makyng

In pe merce of God I haue sayd before

God grawnt ham grace of good endyng

pat done peraftir bop lasse \& more;

\& let ham neuer, lord, be forelore

pat prayn for Jon pe blynd Audlay;

Into pe kyngdam pu ham restore

Vnto pat blis pat lastep fore ay.

Gesegnet seien die, die das wort hören und darnach thun. Alles, was für leib und seele nötig ist, könnt ihr in diesem buche finden. Zum zeugen nehme ich Paulus, den Christus in gnaden aufnahm; so hat er es - glaube ich - auch mit mir gethan, zeit und musse hat er mir gegeben, in meiner blindheit diese worte zn sagen, so grosser stinder ich auch bin. Nehmet euch daher an mir ein beispiel und bessert euch bei zeiten.

Als ich krank war und in thränen lag, glaubte ich einen mann zu hören, der mir sagte: 'Lasse deinen schlaf, denke an gott tag und nacht; denke an das jungste gericht und warne die leute davor.' Als ich erwachte, brach ich in thränen aus und bat Christus, er solle die welt nicht uberraschen mit dem jügsten gericht, sondern sie vorher warnen. Gewähre mir, dass ich ein gutes wort sagen kann.'

Dann beginnt des dichters warnung an seine mitmenschen: 'Ich fürehte, es sieht schlimm mit ihnen aus, sie denken nicht an das göttliche, noch schlimmer aber mit den priestern selbst, die die wahrheit verktundigen sollten, und statt dessen von 
tage zu tage in stinden liegen. Entschuldigt nur nicht eure sünden, das nutzet nichts; beispiel: Adam. - Wer aber hier gebeichtet und busse gethan hat, der wird erlöst. Seid bereit, denn der jungste tag kommt plötzlich wie die sinflut und wie der untergang von Sodom und Gomorrha. Wenn es euch gat geht, habet acht! In krankheit lernt ihr beten. - Ich weiss wohl, dass mich gottes feinde lästern werden, die treue christenleute Lollarden nennen.'

Dann wendet er sich vor allen dingen an die priester, die der gemeinde mit gutem beispiele vorangehen und für sie beten sollen. Und dann wieder: 'Ihr alle, die ihr dies buch höret oder leset, thuet darnach! Ich schmeichele nicht; ich will nur den rechten weg zum himmel zeigen, ausser dem es keinen anderen giebt.

4259 pe cownsel of conseans pis boke I call, Or pe ladder of heuen, I say forewy: per is no mon, may clym vp a walle Without a ladder sekyrly, No more may we to heuen on hye Without treu cownsel of consians.

Clyme vp pis ladder, pen may ze se

What ze schul do to Godis plesans, \& weder ze wil haue wele or wo; Clyme vp pis ladder, pen may ze se,

4270 What ioys in heuen pat per be \& what payns in hel \& turmentre, pen chese zourselue weder to go.

Richtet nicht eure mitmenschen; in eines anderen auge könnet ihr eine einzele mucke sehen, in eurem eignen aber selbst keine neun oder zehn.

Ich danke der heiligen dreieinigkeit, dass sie mich gelehrt hat, vor meinem ende hier auf erden busse zu thun.'

Dann folgen die beiden schlussstrofen, die ich vorne anfuhrte (s. s. 178 f.), und die lateinische schlussbemerkung, die dort gleichfalls steht (8. s. 177).

So weit kam der dichter also im jahre 1426 mit seinen dichtungen, die er unter dem namen 'gewissensrat oder himmelsleiter' zusammenfasste. Aber er hat noch länger gelebt und gedichtet; denn unmittelbar an die schlussbemerkung schliessen sich andere gedichte an. 


\section{Zweite abteilung (4350-5247).}

Erstes (neunzehntes) gedicht (4350-4525).

Das erste gedicht, das neunzehnte der ganzen sammlung und das 28. nach der nummer der handschrift, trägt die tuberschrift: 'Hic incipiunt salutationes beatæ Mariæ virginis'. Fast alle zeilen des ersten teiles dieses gedichtes beginnen mit dem ausrufe 'Hayle'; sie sind zu strofen von zehn zeilen verbunden; die reimstellung ist: $a b a b$ b c b c $d d$; die ersten acht zeilen sind meist viertreffig, zuweilen aber - wie die beiden letzten - stabzeilen; diese beiden bilden den kehrreim jeder strofe und lauten:

Hayle mayd, hayle moder, hayle virgyn swete,

Hayle, grawnt ham pe grace pat pus con pe grete.

Dieses gedicht ist ein gebet an die jungfran Maria in der bekannten schwtllstigen art des mittelalters; seine letzte strofe nennt die funf ${ }^{1}$ freuden der jungfrau. Daran schliesst sich dann unmittelbar der zweite teil an, mit den worten:

$44400 \mathrm{Jesu}$, fore pese ioys $\mathrm{V}$,

0 Jesu, pi moder had of pe.

Das versmass und die strofenform sind dieselben wie im ersten teile, doch beginnen jetzt fast alle zeilen mit ' $O$ Jesu'. Durch diese gleichmässigkeit des zeilenanfanges ist aber der rubrikator verfuhrt worden, die anfangszeichen der strofen ganz falsch und unregelmässig zu setzen; auch hat der schreiber in der vorletzten strofe die zweite zeile des kehrreimes

o Jesu, pi grace \& pi merce,

O Jesu, fore pi moder loue mayde Mary

ausgelassen.

Die letzte strofe (4499 ff.), derer zweite bälfte sich wieder an Maria wendet, hat auch den kehrreim des ersten teiles, während dieser zweite teil im ganzen ein gebet an Jesus ist, der um seiner mutter willen gnade gewähren möge.

Es folgen zwei reimpaare (4509-4512) - mit roter tinte geschrieben -, in denen sich der dichter an gott wendet mit der bitte um gnade für alle, die ihm hier dienen; und dann noch eine zwölfzeilige strofe desselben inhaltes $(\mathbf{a} \mathbf{b}$ a b b a b a a c a c), die den schluss des gedichtes bildet, tubrigens von einer

1 Nach Brandl (Engl. Litteraturgeschichte in Pauls Grundriss II. s. 623) sind es auf dem festlande stets sieben, in England aber stets nur fünf freuden der Maria. 
anderen hand geschrieben zu sein scheint. Dann folgt noch wieder mit roter tinte geschrieben - ein schluss-reimpaar:

4525 Wel ys hym pat wil \& may

4525 a Say pat prayere euery day.

Zweites (zwanzigstes) gedicht (4526-4645).

Das folgende gedicht ist mit dem dritten in der handschrift unter der nr. 29 verbunden. Es trägt die tuberschrift: 'Alia oratio de sancta Maria'. Auch in diesem beginnen die meisten zeilen wieder mit 'Hayle'; aber die strofen sind hier zwölfzeilig mit der reimstellung: $\mathbf{a} \mathbf{b} \mathbf{a} \mathbf{b} \mathbf{a} \mathbf{b} \mathbf{a}$ b b c b c; die zeilen sind viertaktig mit reicher anwendung von stabreimen. Der kehrreim ist einzeilig und lautet:

Hayle blessid froyt, haile swet floure.

Dieses gedicht ist womöglich noch uberschwenglicher als das erste; es schliesst: 'Wenn das jungste gericht naht, sei du unsere haupthilfe, dass wir in den himmel kommen.'

Drittes (einundzwanzigstes) gedicht (4646-4697).

Das dritte gedicht ist uberschrieben: ' $\mathrm{H} \circledast \mathrm{c}$ salutatio composuit angelus Gabriel'; es ist von 4676 ab von einer anderen hand geschrieben. Es sind im ganzen funf zehnzeilige strofen von viertaktigen versen mit der reimstellung $a b$ a b c c de de; die zweite und die vierte strofe sind unregelmässig, haben je eine zeile mehr. Das gedicht erzählt die verktindigung Mariæ and schliesst mit einem gebete an die jungfrau.

Viertes (zweiundzwanzigstes) gedicht (4698-4801).

Das vierte gedicht der zweiten abteilung (30) trägt die therschrift: 'Hic incipit psalmus de Magnificat' und besteht aus 13 achtzeiligen strofen, derer jede mit dem kehrreim schliesst:

Magnificat anima mea Dominum.

Die reimstellung ist: $\mathbf{a} b \mathbf{a} b \mathrm{~b}$ c $\mathrm{b} c$; die verse sind viertreffig.

Die strofe $4746 \mathrm{ff}$. beginnt:

penke on kyng [Robart] Sesel,

He went ne lord had be bot he,

3et sodenle downe he fell

\& was put into a folis degre.

Das Wort 'Robart' ist von anderer hand uber der zeile eingefugt.

'So - heisst es weiter - werden die hohen erniedrigt.'

Der tubrige inhalt entspricht dem gedanken des kehrreimes. 
Funftes (dreiundzwanzigstes) gedicht (4802-5009).

Dieses, nach der handschrift 31., gedicht trägt die tuberschrift: 'Hic incipit salutatio Sanctæ Brigittæ virginis et quomodo dominus Jesus Christus apparait illi corporaliter et dedit illa suam benedictionem suam. quop Awdelay.' Es sind 23 neunzeilige strofen (eine hat durch ein versehen des schreibers eine zeile mehr) mit der reimstellung $\mathrm{a} b \mathrm{a}$ b c d d d c; die ersten vier zeilen sind stabreimzeilen mit sieben treffen, die fünfte und die neunte zeile sind dreitreffig, die sechste bis achte haben je vier takte.

Auch in diesem gedichte beginnt wieder die mehrzahl der zeilen mit 'Hayle'. 'Heil dir, Brigitte, - so fängt es an heil dir als jungfrau, weib und witwe. Du wähltest keuschheit und bestimmtest selbst deinen gatten dazu; deshalb seid ihr beide gesegnet. - Die mutter gottes erschien ihr, auch Christus selbst (4848), oft sprach er mit ihr im gebete. - Heil anch allen, die ihren heiligtums-ort besuchen. - Christus befahl ihr (4857), zum papste Urban nach Rom zu ziehen, um von ihm fur ibre kirche denselben ablass zu erlangen, den auch die besucher der Peterskirche haben. Der papst hat ihre bitte gewährt, als er hörte, dass sie von gott selbst gesandt sei. Nach hause zurtickgekehrt, hat sie dann ein nonnenkloster gebaut, und ihr gatte ein mönchkloster. - König Heinrich V. (4938) griindete 'Bregit Sion' und der papst bewilligte den pilgern ablass; Christus segne ihn dafür. - Gott segne anch den heiligen ort und seine geistlichen, die für uns beten; gott segne auch unseren jungen könig Heinrich VI.'

In der schlussstrofe heisst es: "Alle, die ihr dieses lied leset, betet filr seinen verfasser, der blind und tanb ist, den siindbaften Audelay."

Sechstes (vierundzwanzigstes) gedicht (5010-5131).

Dieses gedicht (nr. 32) hat zur tuberschrift zwei englische zeilen :

5010 Wenefrede pu swete may

Thow pray for vs bope nyzt \& day.

Es besteht aus 30 vierzeiligen strofen von viertreffigen versen mit der reimbindung $a$ a $a$ b; der reim $b$ verbindet alle strofen mit einander. Das gedicht beginnt so: „Du konntest eines flirsten geliebte werden, Wenefrede, aber du wolltest deine 
keuschheit bewahren. Cradoc aber schändete dich und liess dich enthaupten. Dein Oheim Bewno ${ }^{1}$ aber setzte dir dein haupt wieder auf deinen leib, und durch ein wunder lebtest du noch funfzehn jahre. An deinem halse aber konnte man immer die spur des schwertstreiches sehen, und das stand dir ganz gut (5031 Hit besemyd pe wel, sople to say). Cradoc aber wurde nach seinem verbrechen von der erde verschlungen. Dein auf die steine verspritztes blut aber konnte kein wasser wegwaschen, und an der stelle deiner enthauptung entsprang eine quelle. An dem flusse, den diese bildete, stand eine muhle, in deren räderwerk einst ein kind hinein geriet; da stand das wasser still. Als die mutter das kind fand, lachte und spielte es.'

Dann werden noch andere wunder erzählt; $u$. a. dieses: 'Während wein in der kirche lagerte, konnte keiner das wasser der quelle erwärmen; man brachte den wein heraus, da wurde ein mann durch ihn vergiftet, aber durch gottes und der Wenefrede hilfe gerettet. Da lief das wasser, das bisher stille gestanden hatte, weiter. Treibet daher keinen handel in der kirche.'

Am schlusse nennt sich Audelay wieder.

Siebentes (funfundzwanzigztes) gedicht (5132-5199).

Das siebente gedicht ist eine 'Salutatio Sanctæ Wenefredæ virginis'; sie ist wie die der Brigitte in der neunzeiligen strofe mit stabreim gedichtet; derer sind sieben. Auch hier fangen wieder viele zeilen mit 'Hayle' an. 5195-5199 sind funf lateinische zeilen. Das ganze ist ein lobgesang auf die heilige.

Achtes (sechsundzwanzigstes) gedicht (5200-5223).

Dieses gedicht, das die nr. 33 der handschrift hat, ist sehr kurz und trägt die überschrift: 'Quicumque hanc salutationem in honore sanctæ Annæ matris Mariæ quotidie devote dixerit, sine dubio mala morte non morietur.' Es sind nur sechs vierzeilige strofen von stabreimzeilen mit der reimbindung a b a b. Jede zeile beginnt mit dem worte 'Gaude', jede strofe mit 'Gaude felix Anna.'

1 Die handschrift hat bewn on oder bewnou. Beides kann richtig sein, denn Beuno heisst der mann auch in der von Horstmann gedruckten legende (E. E. T. S. 86); von Horstmann wird auch der name Wenefrede erklärt: wen ist wälsch und bedeutet 'bändchen (um den hals)'. 
Hinter dem englischen gedichte folgt ein kurzes lateinisches gebet an die heilige Anna und an gott.

Neuntes (siebenundzwanzigstes) gedicht (5224-5247).

Das folgende gedicht (nr. 34) ist eine 'Salutatio in honore salvatoris'; die lateinische tuberschrift ist sieben zeilen lang und betrifft den ablass, der für die 'Salutatio' gewährt wird.

Darunter befindet sich eine ausserordentlich rohe federzeichnung des schweisstuches der Veronika mit dem kopfe Christi, das einzige bild der handschrift.

Das gedicht selbst besteht aus acht dreizeiligen strofen mit der reimbindung $\mathbf{a}$ a b; b kehrt in je zwei strofen wieder; a ist gleich in der ersten und in der zweiten, dann in der dritten und in der vierten, und schliesslich in den vier letzten strofen; also ein ziemlich kunstvolles gedicht! Die ersten beiden zeilen der strofen sind siebentreffige stabzeilen, die dritte ist dreitreffig.

Den Inhalt bilden die begrtlssung des antlitzes Christi und ein gebet; jede strofe beginnt mit 'Salve'.

Auch auf dieses gedicht folgt wieder ein kurzes lateinisches gebet.

Ueber der nächsten spalte (der zweiten der rückseite des 27. blattes) stehen dann ausser der ur. 35 mit roter tinte geschrieben die beiden zeilen:

5248 I pray zow, syrus, boothe moore \& lase, Syng pese caroles in Cristemas.

Das bezieht sich sicher auf die folgenden kurzen gedichte, derer 25 unter der nr. 35 zusammengefasst sind.

\section{Dritte abteilung (5248-6129).}

Erstes (achtundzwanzigstes) gedicht (5250-5270).

Es trägt die überschrift: 'Hic incipiunt decem pracepta iu modum cantalenæ' und enthält eine mahnung, die zehn gebote zu halten. Es besteht aus einer siebenzeiligen und aus vier funfzeiligen strofen; der kehrreim ist 'Leue ze me'; die anderen zeilen sind viertaktig und haben die reimbindung a b a b; die erste strofe bat noch ein reimpaar davor. 
Zweites (neunundzwanzigstes) gedicht (5277-5303).

Dieses gedicht handelt 'De septem peccatis mortalibus'. Seine äussere form ist genau dieselbe wie die des vorigen gedichtes; der kehrreim lautet: 'I say pe so'. - Der dichter giebt den rat, die sieben todsünden zu meiden, und empfiehlt für jede ein gegenmittel, demut gegen neid, keuschheit gegen unzucht usw.

Drittes (dreissigstes) gedicht (5304-5330).

'De septem opera misericordiæ' lautet die aufschrift dieses gedichtes, das wieder dieselbe form hat wie die beiden vorigen, aber mit dem kehrreime: I cownsel pe. - Den inhalt bildet die mahnung, die sieben werke der barmherzigkeit zu üben.

Viertes (einunddreissigstes) gedicht (5331-5356).

Dieses gedicht, 'De quinque sensus', hat wieder dieselbe form; aber der kehrreim (Lest pou be chent) fehlt bei der letzten strofe. Hier mahnt der dichter, die funf sinne recht zu gebrauchen.

Füntes (zweiunddreissigstes) gedicht (5357-5388).

Das füfte gedicht handelt 'De septem dona spiriti sancti'; die form ist wieder dieselbe wie bei den vorigen gedichten, doch hat dieses noch eine sechste strofe. Der kehrreim lautet: 'Ellis were we lost'. - Audelay ermahnt hier, die sieben gaben des heiligen geistes richtig anzuwenden. - In diesem wie in den vorhergehenden vier gedichten finden sich viele, z. t. wörtliche wiederholungen aus dem grossen zweiten gedichte der ganzen sammlung.

Sechstes (dreiunddreissigstes) gedicht (5389-5410).

Die uberschrift dieses gedichtes lautet: 'In die natalis domini'. Es besteht aus einer sechs- und vier vierzeiligen strofen; diese haben die reimbindung a a ab, in jener geht noch ein reimpaar voraus. Alle zeilen sind viertreffig und beginnen mit ausnahme von zweien mit dem worte 'Welcum'; der kehrreim lantet: 'Welcum zole for euer \& ay'. Der inhalt ist kurz: 'Willkommen seid mir: Weihnachten, Christus, Maria, Stephan, Johannes, Thomas, Neujahr, 12 Nächte. - Seid fröhlich beim weihnachtsfeste.' 
Siebentes (vierunddreissigstes) gadicht (5411-5432).

Dieses gedicht ist tiberschrieben: 'In die Stephani'; es hat dieselbe form wie das vorige, doch kommt kein kehrreim vor; die letzten zeilen der strofen reimen aber unter einander. - Inhalt: 'Verehret den heiligen Stephan, den ersten Märterer.

Achtes (funfunddreissigstes) gedicht (5433-5474).

'In die sancti Iohannis' ist das nächste gedicht, das wieder dieselbe form hat wie das vorige, aber der vierzeiligen strofen sind neun. Die schlussstrofe entspricht bis auf den namen des heiligen wörtlich der des vorigen gedichtes. Inhalt: 'Dem Johannes hat der herr beim scheiden seine mutter anvertraut. Sei du, heiliger Johannes, unser fürbitter.'

Neuntes (sechsunddreissigstes) gedicht (5475-5500).

Dies gedicht ist 'In die sanctorum Innocentium'; die form ist dieselbe wie bei den vorigen gedichten; es hat eine sechs- und funf vierzeilige strofen. - Der anfang ist von eigentumlicher naturwtichsigkeit:

5475 With al pe reuerens pat we may

Worchip we childermasday.

Crist crid in cradil 'moder, baba'

pe childer of I[s]ral cridyn 'wa wa',

Fore here merb hit was aga,

5480 When Erod fersly cowp hem tray. lehem.

Das gedicht erzählt im ubrigen den kindermord zu BethZehntes (siebenunddreissigstes) gedicht (5501-5522).

'De Sancto Thome Archiepiscopo Cantuariensi' lautet die tuberschrift des nächsten gedichtes. Form: Wie bei den vorigen. Funf strofen. - Kurze geschichte des heiligen Thomas.

Elftes (achtunddreissigstes) gedicht (5523-5562).

Dieses elfte gedicht trägt die aufschrift: 'In die circumcisionis domini'. Es umfasst 20 zeilen, die sich, wie sie uberliefert sind, nicht in strofenform bringen lassen; in der mitte findet sich ein leerer raum, wo etwa vier zeilen weggeschabt sind; auch sind hier verschiedene zeilen, sowie einzele wörter und buchstaben von einer anderen hand geschrieben. Auch der inhalt ist nicht recht klar. 
Zwölftes (neununddreissigstes) gedicht (5563-5628).

Das zwölfte gedicht ist ein weltliches; es handelt 'De rege nostro Henrico sexto'. Die form ist genan dieselbe wie beim siebenten bis zehnten gedichte dieser abteilung; es sind eine sechszeilige und funfzehn vierzeilige strofen.

Inhalt: 'Gott, wir bitten dich: beschtttze unseren könig tag und nacht, denn er ist sehr zarten alters. Seine ahnen alle waren königlichen geschlechtes. Sein vater fthrte krieg mit Frankreich aus liebe zur prinzess Katharine; 'Harflet' hat er belagert, bei 'Agyncowrt at pat patayle' hat er die Franzosen besiegt. Dann heiratete er Katharinen, und sie gebar ihm den jetzigen könig. An diesem wird sich die weissagung erfullen, dass er das heilige kreuz vor seinem tode gewinne und die ganze heidenwelt tanfe.' Die schlussstrofe lautet:

56253 if he fare wele, wele schul we be,

Or ellis we may be ful sore;

Fore him schal wepe mone an e.

pus prophecis pe blynd Awdlay.

Dieses gedicht giebt uns einen neuen anhaltspunkt fur die bestimmung der entstehungszeit der gedichte. Da die schlacht von Azincourt (1415) erwähnt wird, und es von könig Heinrich VI., der 1421 geboren wurde und 1422 zar regierung kam, heisst: 'he is ful zong, tender of age' (5565), so kann das gedicht nur nach dem jahre 1422 entstanden sein.

Dreizehntes (vierzigstes) gedicht (5629-5670).

Dieses gedicht hat die therschrift 'Fac ad quod venisti'. Es besteht aus einer sieben- und sieben funfzeiligen strofen mit der reimbindung $\mathbf{a} \mathbf{b} \mathbf{a} \mathbf{b} \mathbf{c}$; die erste strofe hat noch ein reimpaar voraus. Die letzte zeile jeder strofe lautet: 'I say allgate vt supra'; da aber auch schon die der ersten diesen wortlaut hat, so ist nicht klar, wa s zu ergänzen isr.

Inhalt: 'Schuster, bleib bei deinem leisten. Wozu euch gott im leben bestimmt hat, das thuet! Vier dinge hasst gott: Einen harten priester, einen stolzen bruder, einen unztichtigen alten mann, einen feigen ritter'. Dann folgt, was die einzelnen thun sollen.

Vierzehntes (einundvierzigstes) gedicht (5671-5680 und 5868-5891).

Dieses gedicht ist eine 'Cantalena de pueritia'; es besteht aus einer sechs- und sieben vierzeiligen strofen mit der 
reimbindung $a \mathrm{a} a \mathrm{~b}$ und in viertaktigen zeilen; $\mathrm{b}$ ist in allen strofen gleich. Mit vers 5680 endigt das 29. blatt; die folgenden beiden blätter ( 30 und 31 ) mulssen umgelegt werden, denn auf dem 31. geht unser gedicht weiter. Ich behalte aber die verszahlen nach der handschrift bei, da ich in dieser durchgezählt habe.

Inhalt: 'Wenn gott mir meine bitte gewähren wollte, so möchte ich wieder ein kind sein, dem kirschensteine ein schatz sind. .... Es hasst alle todstinden. Ich aber fiel in stinden. 0 wäre ich doch gestorben, als ich ein kind war; ich sehe hier kein anderes heil als busse zu thun.'

Funfzehntes (zweiundvierzigstes) gedicht (5892-5936).

Dieses gedicht ist 'In die Epephaniæ'; es besteht aus elf vierzeiligen strofen mit der reimbindung $a b$ a $b$. Ueber der ersten strofe steht: 'Nowel, nowel, nowel'. Die vierte zeile jeder strofe ist lateinisch.

Das gedicht erzählt die geschichte der heiligen drei könige.

Sechszehntes (dreiundvierzigstes) gedicht (5937-5983).

'De sancta Anna matre Mariæ' lautet die uberschrift dieses gedichtes. Die form ist genau dieselbe wie bei den ersten dieser abteilung, die zahl der strofen ist neun; der kehrreim lautet: 'Herefore we (oder: I) say'. Die schlussstrofe ist halb englisch, halb lateinisch. Der inhalt des gedichtes ist im wesentlichen derselbe wie der des fruheren (sechsundzwanzigsten, achten der zweiten abteilung, s. s. 204 u.).

Siebenzehntes (vierundvierzigstes) gedicht (5984-6029).

Die uberschrift lautet hier: 'Alia cantalena de Sancta Maria'. Auf drei einleitungszeilen folgen sieben sechszeilige strofen mit der reimbindung a a a $\mathrm{b} b$; die ersten vier und die letzte zeile haben vier takte, die funfte aber nur zwei. In diesem liede wird Jesus mit einer blume verglichen, Mariæ verkundigung und Jesu geburt gepriesen.

Achtzehntes (funfundvierzigstes) gedicht (6030-6057 und 5681-5694).

Noch ein Marien-lied: 'Et alia de sancta Maria'. Es besteht aus zwei einleitungszeilen und funf achtzeiligen strofen 
mit der reimbindung $\mathrm{a} b \mathrm{a} \mathrm{b} \mathrm{cd} \mathrm{ed}$; die letzten zeilen sind lateinisch. Jede strofe beginnt mit: 'Gaude Maria'. Von der vierten strofe schliessen die beiden ersten zeilen das 31 . blatt.

Die folgenden zeilen stehen auf dem 30. (vgl. s. 209 o.). Ein lobgesang auf die jungfrau.

Neunzehntes (sechsundvierzigstes) gedicht (5695-5716).

'De sancta Maria' steht tuber diesem gedichte; es besteht aus einer sechs- und aus vier vierzeiligen strofen in der art der fruberen mit der reimbindung $\mathbf{a} a \mathbf{a} \mathbf{b} ; \mathbf{b}$ verbindet wieder die einzelnen strofen; ein reimpaar $b$ b eröffnet das gedicht. Jede strofe ausser der zweiten beginnt mit 'Hayle'. Es ist ein lobgedicht auf die jungfrau, die auch als 'chif chosun garbunkulston' angeredet wird (5709).

Zwanzigstes (siebenundvierzigstes) gedicht (5717-5741).

Dieses gedicht, mit der aufschrift: 'De virginitate', preist die jungfräulichkeit. Die form ist dieselbe wie beim vorigen gedichte. Hier sind wieder mehrere zeilen und einzele wörter von anderer hand geschrieben; auch sind hinter der ersten zeile der dritten strofe (unten an der seite), sowie hinter der vierten und hinter der funften strofe je zwei leere zeilen.

Einundzwanzigstes (achtundvierzigstes) gedicht (5742-5775).

Dieses ist eine 'Cantalena de virginibus'. Inhalt wie beim vorigen; form wie bei den beiden vorhergehenden; acht strofen (davon eine, die erste, mit sechs zeilen).

\section{Zweiundzwangstes (neunundvierzigstes) gedicht} $(5776-5812)$.

Das folgende gedicht handelt: 'De matrimonio mulierum'. Es hat dieselbe form wie die ersten funf, das dreizehnte und das sechszehnte dieser abteilung, aber eine strofe weniger (sieben); der kehrreim lautet: 'All day pou seest'. Dieses gedicht bringt eine, oft wörtliche wiederholung dessen, was schon in den allerersten gedichten gesagt wurde; also hier lob und heilighaltung der ehe. 
Dreiundzwanzigstes (funfzigstes) gedicht (5813-5838).

Dieses gedicht heisst 'De amore Dei'. Es hat genau dieselbe form wie das vorige gedicht, aber nur funf strofen; der kehrreim lautet: 'I say herefore'. - Inhalt: 'Gott erwartet fur seine liebe nur gegenliebe.'

Vierundzwanzigstes (einundfunfzigstes) gedicht (5839-5867 und 6058-6074).

Dieses gedicht trägt die uberschrift: 'Tim or mortis conturbat me'. Es besteht aus elf strofen von vier viertreffigen zeilen mit der reimbindung $a b a b$; die erste strofe hat noch ein reimpaar vorher. Der kehrreim lautet: 'Passio Christi, conforta me'. Die dritte zeile der siebenten strofe ist die letzte des 30. blattes, dann geht es auf dem 32. weiter (vgl. s. 209 o.).

Inhalt: 'Todesfurcht verwirret mein herz; ich bin blind, taub und krank. Aber gottes wille geschehe! Sein name sei gesegnet.'

Der dichter wiederholt sich dann wieder und nennt am schlusse seinen namen.

Fünundzwanzigstes (zweiundfunfzigstes) gedicht (6075-6129).

Dieses letzte gedicht der dritten abteilung handelt 'De sancto Francisco'. Es besteht aus einer sieben- und zwölf vierzeiligen strofen mit der reimstellung a a a b; b verbindet die einzelen strofen untereinander; die erste strofe hat die reimbindung (mit ricksicht auf die folgenden bezeichnet) $\mathrm{b} b$ a a a b b.

Der dichter erzählt hier die geschichte des heiligen Franz und nennt sich wieder am schlusse.

\section{Vierte abteilung (6130-6354).}

Hinter der letzten zeile des vorigen gedichtes folgen vier leere zeilen und dann gleichsam als tuberschrift zum folgenden:

6130 Rede thys offt, butt rede hit sofft,

And whatt pu redust, forzeete hit nozt;

For here pe soth pou mazht se,

What fruyte comep of py body.

Dann folgt die eigentliche tuberschrift: 'De peccatis cordis' und eine lange abhandlung, vom rubrikator als nr. 36 bezeichnet. Sie umfasst 221 zeilen. Audelay sagt zuerst: 'Die 
stinden des herzens sind folgende' und dann zählt er sie auf (6134-6161); dann fthrt er 'die stunden des mundes' auf (6162-6180) und endlich 'die stinden der that' (6181-6202); dann nennt er die 'umstände' der stinde (6203-6242). Mitten in der prosa stehen einmal funf zeilen in der art des zweiten teiles der auch sonst von Audelay verwendeten dreizehnzeiligen strofe (6224-6228).

Hinter 6242 kommen wieder vier leere zeilen und dann beginnt ein neuer teil, der kurz folgenden inhalt hat: 'Wenn du die kammer deiner seele von aller sünde reinigst, so sollst du aus den gedanken an deine studen eine strohunterlage machen, aus der du den staub aller sünde und alle bösen gedanken mit der 'schakeforke of kyndnes' entfernen musst. Darauf lege dann als matratzen die sorge um deine sunde und die nachdenklichkeit. Die beiden betttucher sollen sein enthaltsamkeit und keuschheit, usw. usw. Die kopf kissen seien mitleid und geduld, die polster wachsamkeit und geschäftigkeit, der rechte vorhang recht und vernunft, der linke verstand und weisheit. Diese vorhänge sollen an den ringen der zehn gebote laufen; wenn ein ring zerbricht, fallen die vorhänge herab, und der teufel kann dann in dein bette sehen; usw. usw.' Am schlusse heisst es: 'This is pe bed pat our lord spekep of in pe boke of lawe Canticorum primo : lectulus noster iam floridus est' - und weiter: 'Thus is pe bedde pat our lord wil haue lykyng to ly in, \& when he is in pis bed, angelys wil syng about him pis song of prophese : Exulta \& lauda, habitatio Syon."

\section{Fünfte abteilung (6355-6657).}

Erstes (dreiundfunfzigstes) gedicht (6355-6424).

Dieses gedicht hat in der handschrift die nummer 37 und die uberschrift 'Pater noster' qui es in cælis'. Es besteht aus sieben strofen, deren jede eine der sieben bitten des vaterunsers behandelt und auch ihren lateinischen text in der drittletzten zeile einfugt. Die strofe hat elf zeilen und die reimstellung $a b a b a b a b c d d$; besonders verwickelt und schwierig wird diese reimart noch dadurch, dass in je zwei der je acht ersten zeilen nur die selbstlauter verschieden, die mitlauter aber gleich, die schliessenden sogar alle gleich sind, 
z. b. prise : prayse : ryse : rayse : asyse : sayse : yse : ayse und fode : fede : houyd : hede : blood : blede : rode : rede. - Ausser der lateinischen, sowie der vorletzten zeile, die nur drei takte bat, sind alle zeilen siebentaktig, und zwar sind es offenbare stabzeilen, wenn auch die stäbe oft sehr unregelmässig gesetzt sind. Als probe gebe ich die erste strofe:

6355 The páter nóster tó expóne máy no mán hit príse, pát of práyérs is prís \& móst fóre to práyse. I réde pou réde hít arýzt \& out of sýn rýse, pát may réstýng in héuen vntó pi sóule ráyse. fure séuen póyntís per béne esét ín a sýse,

6360 pe léstys sálue is tó pe sýn, ás pe bóke sáyse.

Oure fáder pe wýche bou hárt in héuen, pis óresóne pou jise. Áy o[-]hálouyd bé pi náme in ángyr \& in áyse!

Sanctificetur nomen tuum!

Sáy whé pe sáme:

Oure fáder pe wýche pou árt in héuen, hálouyd bé pi náme!

\section{Zweites (vierundfunfzigstes) gedicht (6425-6565).}

Unter der nächsten nummer der handschrift (38) sind dio drei letzten gedichte der ganzen sammlung zusammengefasst.

Das erste von ihnen trägt die uberschrift: 'De tribus regibus mortuis' und besteht aus elf dreizehnzeiligen strofen, die anders gebaut sind als die der ersten gedichte; die reimbindung ist $\mathbf{a} b \mathbf{a} b \mathbf{a b} a b c d a c d$. Noch deutlicher vielleicht als in dem vorigen gedichte liegt in diesem die alte stabzeile vor; als probe gebe ich die erste strofe.

6425 An a býrchýn bónke, per bóus árne brýzt,

I sáw a brýmlýche bóre tó abáy brózt,

Rónke ráchís with rérde, pai rónnón arýzt,

Of àl hore rów \& hóre rést lýtil hom pózt.

Me pózt hít ful sémelé to sé sóche a sézt,

6430 How in a sýde of a sálze a séte hím he sózt

Fró pe nóyse pat hít was nêw til hít was né nýzt,

Fro pe nón bót a nápwíle; me pózt hít bot nózt.

Me pozt hit nozt bot a prow,

To se, how he probyt \& prew.

6435 Honters with hornes pai kowp blow, pai halowyd here howndys with how;

In holtis herde I neuer soche hew.

Auch hier haben wir also die beim vorigen gedichte besprochene kunstvolle reimart, und zwar nicht allein im ersten teil, sondern auch, allerdings gesondert, im zweiten teil. 
Ich habe diese stabzeilen von anfang an in diesem anfsatze siebentreffig genannt; schon unabhängig von professor Trautmann war ich nämlich bei der prüung der metrik Audelays zu der tuberzengung gelangt, dass sich Audelays stabzeilen am besten und flussigsten mit sieben treffen lesen lassen; jetzt sebe ich zu meiner frende meine ansicht bestätigt, die professor Trautmann in seinem aufsatze auf s. 83-100 dieses bandes fur die mittelenglische stabzeile tuberhaupt aufstellt. Je mehr ich jetzt mittelenglische stabzeilen mit vier and mit sieben takten zu lesen versuche, je mehr scheint mir unsere ansicht die richtige, dass sie mit sieben treffen gelesen werden mussen.

Eigentumlich ist der inhalt dieses gedichtes: 'Ich sah einen eber von hunden gestellt; drei könige kamen dazu und erzählten sich geschichten. Ein nebel uberfällt sie, und geister erscheinen; diese hält der erste könig für geister, der zweite fur frauen, der dritte für zauberer; alle drei aber haben grosse angst vor dem spuke. Da spricht der erste der geister: 'Wir sind eure väter', und ermahnt sie.' Diese verse sind so schwer verständlich, dass ich einstweilen nur den ungefähren sinn herauslesen kann. 'Die könige kehren heim und bauen ein muluster.'

\section{Drittes (funfundfunfzigstes) gedicht (6566-6605).}

Die uberschrift dieses gedichtes lautet: 'Non honor, sed (h)onus assumere nomen honoris'. Es ist das vierzigzeilige lateinische gedicht 'Cur mundus militat', das sich schon in Wrights ansgabe der 'Latin poems' (Camden Society. 16. band. 8. 147) des Walter Mapes findet.

Dieses gedicht ist von einer ganz anderen hand geschrieben, die aber nach Mr. Madans ansicht nur wenig später ist als die frthere.

Viertes (sechsundfunfzigstes) gedicht (6606-6657).

Auf dem letzten blatte der handschrift (dem 35.) steht das letzte gedicht der ganzen sammlung, das wieder von einer anderen hand geschrieben ist, die aber auch schon fruher vorgekommen ist, und die gleichfalls nicht viel später ist.

Dieses gedicht besteht aus vier dreizehnzeiligen strofen in der art der frtheren. Die therschrift lantet: 'Sapientia huius mundi stultitia est apud deum'. Der inhalt ist: 'Hier könnt ihr hören, was ihr seid und was die welt ist, hier erfahren, 
dass kein anderer gott ist als der herr, der alle dinge durch e in wırt geschaffen bat. Wir sind geboren, dem allmächtigen zu dienen und seine gebote zu halten. Gebet daher nichts anf die frenden dieser welt, wo alles eitel ist. Denket, wie balde ibr sterben könntet.' Ueber der dritten strofe steht: 'Hic vir despiciens mundum', und in ihr sagt der dichter dann: 'Die welt habe ich verachtet and mir den himmel durch wort und that erkauft, indem ich in meinem leben den anderen mit gutem beispiele voraufging. In diesem buche könnt ihr meinen willen erkennen. Thuet busse, ehe ihr aus dieser welt scheidet; denn gut ist alles, was ein gutes ende nimmt; so rät euch der blinde John Audelay.' Dann folgen die zeilen:

Cuius finis bonus, ipsum totum bonum.

Finito libro sit laus et gloria Christo!

und darauf die schlussstrofe, die schon oben (s. 179 u.) abgedruckt ist.

Es scheinen noch ungefähr 20 zeilen beschrieben gewesen zu sein, aber alle schrift ist weggeschabt; ein langer breiter tintenstreifen befindet sich in der mitte dieser zwanzig zeilen, wahrscheinlich mit dem finger hingeschmiert, um ein paar zeilen recht grtindlich za verdecken.

Auf der rtlckseite des blattes sind noch allerhand kritzeleien; ganz zu entziffern ist unter ihnen nur folgendes verspaar:

Vertitur in cineres flos fratrum, flent mulieres,

Hic jacet Andreas, qui lapidsvit eas.

\section{Uebersicht über die von Audelay verwendeten strofenformen.}

\begin{tabular}{cll} 
Zahl der zeilen. & \multicolumn{2}{c}{ Reimbindung. } \\
13. & a b a b b c b c d e e d & $1.2 .^{*} 3.11 .15 .16 .18 .56$. \\
13. & a b a b a b a b c d c c d & $54 .^{*}$ \\
12. & a b a b b a b a a c a c & 19 (die schlussstrofe). \\
12. & a b a b a b a b b c b c & 20. \\
11. & a b a b a b a b c d d & $53 .^{*}$ \\
10. & a b a b b c b c d d & 19. \\
10. & a b a b c c d e d e & 21. \\
9. & a a a a b c c c b & $14 .^{*}$ \\
9. & a b a b c d d d c & $23 .^{*} 25 .^{*}$ \\
8. & a a a b c c c b & $8^{*}($ die mittleren $7(8)$ strofen). \\
8. & a b a b b c b c & 13.17 .22$. \\
8. & a b a b c d c d & 45.
\end{tabular}

* Die so bezeichneten strofen bestehen zum grössten teile sus stabreimzeilen. 
Zahl der zeilen.

6.

6.

5.

4.

4.

3.

Reimpase.
Reimbindung. a a b c c b

\&\& \& \& b b

\& b \& b c

a \& $\& b$

$\mathrm{ab}$ a b

a $\mathbf{a}$ b
Nr. der gedichte.

4. 5. 7. 8 (die erste und die drei letzten strofen). 9. 12.

44.

28-32. 40. 43. 49. 50.

24. 33-37. 39. 41. 46-48. 52 .

26.* 42. 51 .

27.*

10.

Die verschiedenen metrischen formen behandelt Audelay mit leidlichem geschick, selbst diejenigen, allerdings nicht zahlreichen, die ziemlich kunstroll sind. Seine stoffe sind ja nicht nen, aber aus der behandlung aller blickt doch der von tiefer reue tuber die frtheren sünden durchdrungene sinn des blinden mannes hervor, der alles versucht, seine mitchristen zam guten zu erziehen, vor allem aber die priester an ihre pflichten zu mahnen, wie es um dieselbe zeit oder etwas später, gleichfalls in Shropshire, auch John Myre in seinen 'Instructions for Parish Priests' that, die Edward Peacock 1868 fur die E. E. T. S. herausgegeben hat.

\section{Anhang.}

Die dreizehnzeiligen strofen mit stabzeilen im Mittelenglischen.

In seinem 'Grundriss der englischen Metrik' (Wien u. Leipzig 1895) sagt Schipper auf s. 92: „Ausserdem (nämlich ausser Golagros \& Gawayn, The Buke of the Howlat, Rauf Coilzear, The Awntyrs off Arthure at the Terne Wathelyne, The Pistill of Susan und Douglas' Prolog zum 8. buche seiner Eneïde) gehören hierher (d. b. zu den alliterierend-reimenden dreizehnzeiligen strofen) noch die im 15. jh. in Sudengland (! muss heissen Shropshire) entstandenen gedichte John Audelays (Percy Society, XIV, s. 10 ff.), ferner das im norden, in 14-zeiligen strofen geschriebene gedicht 'Of Sayne John the Evangelist' (E. E. T. S. 26, p. 87). Die strofen dieser gedichte bestehen allgemein ausgedruckt - aus zwei ungleichen teilen, wovon der erste, längere, der aufgesang, sich in alliterierenden, kreuzweise reimenden $(a b a b a b a b)$ langzeilen bewegt, während der zweite, kturzere, der abgesang, funf oder sechs verse umfasst, deren erster entweder gleichfalls eine langzeile, oder, wie in The Pistill of Susan, ein kurzer aus einer hebung und 
den dazu gehörigen senkungen bestehender vers ist, woranf dann vier zweihebige halbverse folgen. Nur in dem zuletzt genannten gedicht besteht der abgesang aus sechs zweihebigen halbversen." Schipper hätte hier erwähnen sollen, dass bei Audelay in dem ihm bekannten gedichte die reimstellung des Aufgesangs a b a b b c b c ist, weil diese reimbindung in dieser strofenform sonst nicht vorzukommen scheint (vgl. s. 188).

Besondere erwähnung verdient jedes falles die thatsache, dass die dreizehnzeilige strofe mit stabzeilen noch zu anfange des 15. jahrhunderts in Shropshire vorkommt, während bisher im wesentlichen nur nördlichere beispiele bekannt waren (vgl. auch Trautmann, Anglia II, 408).

Ich stelle die verschiedenen formen dieser dreizehnzeiligen stabreimstrofen hier einmal thbersichtlich zusammen:

1. $a b$ a b a b a b c d d d c

$$
\text { Stabzeilen } \underbrace{22}_{*} 2^{* *}
$$

2. $a b$ a b a b a b c d d d $c$

$$
\underbrace{}_{\text {Stabzeilen }} \underbrace{222}_{*} 2^{* *}
$$

* Nach Trautmann (Anglia II, 408 o.) d re i treffig, nach demselben (Anglia XVIII, $90 \mathrm{ff}$.) vi e r treffig.

** Nach Trautmann (Anglia XVIII, 90) dreitreffig.
3. $a b a b a b$ a b cd c c d Audelay nr. 54.

\section{Stabzeilen 4treffig (unregelm.)}

4. $a$ b a b b c b c d eeed Audelay nr. 2.

$$
\text { Strbzeilen } 34443
$$

Man vergleiche noch folgende strofenarten:

14 zeilen: a

$$
\underbrace{a \text { b a b a b a b }}_{\text {Stabzeilen }} \frac{c c d \text { c cd }}{\text { 2treffig (Schipper) }}
$$

12 zeilen : a b a b a b a b cd cd Stabzeilen.

11 zeilen: $\underbrace{a \text { b a b a b a b }}_{\text {Stabzeilen }}$

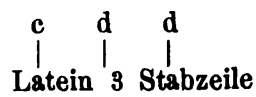

Bons, im September 1895.
St. John.

Song of the Husbandman.

Audelay nr. 53.
J. ERNST WÜLFING. 